\title{
Removal of the deposition on JT-60 tile by nano-second pulsed-laser irradiation
}

\author{
K. Sugiyama ${ }^{*}$, Y. Sakawa ${ }^{2}$, T. Tanabe ${ }^{1}$, D. Watanabe ${ }^{3}$, T. Shibahara ${ }^{3}$ and K. Masaki ${ }^{4}$ \\ ${ }^{1}$ Interdisciplinary Graduate School of Engineering Sciences, Kyusyu University, \\ Fukuoka, 812-8581, Japan \\ ${ }^{2}$ Institute of Laser Engineering, Osaka University, Yamadaoka, Suita, 565-0871, Japan \\ ${ }^{3}$ Graduate School of Engineering, Nagoya University, Chikusa-ku, Nagoya, 464-8603, Japan \\ ${ }^{4}$ Japan Atomic Energy Agency, Mukouyama 801-1, Naka, Ibaraki 311-0193, Japan
}

\begin{abstract}
To clarify the property of tritium removal from carbon codeposition by using pulsed-laser induced desorption, hydrogen removal from co-deposits on JT-60 divertor tile using a fourth-harmonic emission $(266 \mathrm{~nm})$ of a nano-sec Nd:YAG laser was demonstrated. The threshold laser fluence for ablation was $0.3 \mathrm{~J} / \mathrm{cm}^{2}$, which was slightly higher compared to that of the pico-sec laser irradiation. The energy absorption coefficient for the nano-sec laser, which was obtained by fitting the removal rate by so-called Beer's law, was larger than the pico-sec laser. Since the time constant of thermal wave propagation into the target is of the order of nanosecond, such differences between nano- and pico-sec lasers could be attributed to thermal effects. The ablation threshold of the deposited layer was lower than that of a pure graphite, which could be attributed to the difference of thermal conductivity between deposited layer and pure graphite. The removal rate of the nano-sec laser was higher than that of pico-sec laser in the fluence range of $<0.5 \mathrm{~J} / \mathrm{cm}^{2}$. On the other hand, the production ratio [hydrocarbon species $] /\left[\mathrm{H}_{2}\right]$ continuously increased with the laser fluence, and no significant ionization of carbon was observed in this fluence range. This indicated that the fluence range in this study was "weak"-ablation range, which was still not sufficient to minimize the hydrocarbon production.
\end{abstract}

PACS No: 28.52.-s, 79.20.Ds, 79.20.La

Keywords: Laser ablation, Carbon-based material, Tritium

\section{Corresponding author: Kazuyoshi SUGIYAMA}

Interdisciplinary Graduate School of Engineering Sciences, Kyushu University, Hakozaki 6-10-1, Higashi-ku, Fukuoka, Japan

(*Present affiliation: Max-Planck-Institut für Plasmaphysik, EURATOM Association,

Boltzmannstrasse 2, Garching, Germany)

TEL/FAX: +49 (0)89-3299-1492 / +49 (0)89-3299-1212

E-mail: kazuyoshi.sugiyama@ipp.mpg.de 


\section{Introduction}

Carbon based material is currently favored plasma facing material (PFM) because of its good thermomechanical properties and less impact to the core plasma. The present ITER design is planning to use carbon fibre composite as the divertor target which is exposed to the highest heat load in the plasma facing wall [1]. On the other hand, the major disadvantage for the use of carbon as PFM is high erosion yeild due to the chemical sputtering by hydrogen and subsequent carbon-tritium codepostion formation. Particularly, codeposition in plasma-shadowed area is one of the critical concerns because it's difficult to remove it by general plasma-discharge cleaning methods, meaning this can cause continuous tritium accumulation in the vacuum vessel. From the safety reason, periodic tritium removal will be required before in-vessel tritium inventory reaches the administrative goal of ITER (700 g) [2]. In this respect, removal efficiency of the codeposition in shadowed area is one of the key issues for ITER operation program.

Laser induced desorption (LID) is a promising method for tritium removal from the codeposition. Especially, it is expected to be useful for the removal of codeposition in plasma-shadowed area because it is technically practicable by using robotically controlled laser system. There are two major concepts in the application of LID. One is heating the deposition surface up to $>1000{ }^{\circ} \mathrm{C}$ by continuous-wave laser irradiation [3,4]. In this case tritium is thermally desorbed from the codeposition without production of hazardous tritiated debris. Although this method is effective for tritium retained in the near surface, complete removal from thick codeposited layer is difficult. Another approach is the ablation-assisted removal by using high-power pulsed-laser irradiation [5-12], which is the topic of this paper. It is possible to remove not only tritium but also deposited layer itself by ablation, hence almost complete removal is expected. In this method, however, some fraction of tritium can be released as hydrocarbon species, which is undesirable from the viewpoints of tritium processing in the fuel cycling or its biological influence. Nevertheless, since ablation properties depend upon irradiation condition, it is possible to minimize such disadvantage by optimizing the laser parameters. 
In the previous work, we investigated effects of laser wavelength on ablation of codeposition using the fundamental $(1064 \mathrm{~nm})$ and the forth-harmonic $(266 \mathrm{~nm})$ emission of Nd:YAG laser with 20 pico-sec pulse duration [12]. According to the result, the laser irradiation with short-wavelength (266 $\mathrm{nm})$ showed better LID property. It was because the energy of $266 \mathrm{~nm}$-photons $(4.7 \mathrm{eV})$ was more suitable for cutting $\mathrm{C}-\mathrm{H}$ bond whose binding energy is $\sim 4.5 \mathrm{eV}$ and ionization of carbon $(11.3 \mathrm{eV})$ by three-photon ionization. In addition to the wavelength, the pulse duration can play an important role because the effect of thermal wave propagation changes depending on the pulse duration. From this point of view, the ablation behavior using a longer pulse-duration (nano-sec) laser was investigated in this study and compared to the previous results.

\section{Experimental procedure}

The sample used in this study was a JT-60 inner divertor tile, which was exposed to 1800 hydrogen discharges from June 1988 to October 1988 including 1500 discharges of the limiter configuration and 300 discharges of the lower X-point divertor configuration. The tile surface was covered by carbon deposited layer with the thickness of $35-40 \mu \mathrm{m}$. According to a past investigation, hydrogen concentration in the deposition on the JT-60 inner divertor was nearly constant over the whole layer with the $\mathrm{H} / \mathrm{C}$ ratio of $\sim 0.03$ [13]. An isotropic graphite sample (ETP-10) was also prepared for comparison.

The detail of experimental setup is figured in ref. [10]. A small piece with the dimension of $\sim 30 \times$ $10 \times 1 \mathrm{~mm}^{3}$ was cut out from the plasma facing surface of the tile, and situated in the vacuum chamber (background pressure $<3 \times 10^{-8}$ Torr). A Nd: YAG laser (Continuum Minilite) with the maximum laser energy of $\sim 3 \mathrm{~mJ} /$ pulse was focused on the target surface by a quartz lens through a quartz window with a normal direction to the target. The wavelength was the fourth harmonic emission of Nd: YAG laser, i.e. $266 \mathrm{~nm}$, and its pulse duration was $\sim 4$ nano seconds. 
Emitted ions and desorbed gases were measured by time-of-flight mass spectrometer (TOFMS, scan range: $m / e 1 \sim 300$, scan speed: $\sim 0.1 \mathrm{sec} / \mathrm{scan}$ ) and quadrupole mass spectrometer (QMS, scan range: $m / e=1 \sim 80$, scan speed: $\sim 0.5 \mathrm{sec} / \mathrm{scan})$, respectively. Visible-light emission was monitored by a spectrometer (Hamamatsu C7473).

The laser fluence was varied in most cases by changing the focal-spot size on the target. 1000 shots of the pulsed laser beam were irradiated on the target with $10 \mathrm{~Hz}$ of repetition rate. After the laser irradiation, the laser-spot size was measured by scanning electron microscope and the ablation depth was determined by an optical microscope measuring the deference of focal lengths between the surface and the bottom of ablation hole.

\section{Result \& Discussion}

\subsection{Ablation depth}

Fig. 1 shows the ablation depth per pulse obtained from each sample as a function of laser fluence. For the analysis, following two different equations are applied to the experimental data:

$$
\begin{aligned}
& \Delta d \sim \alpha^{-1} \ln \left(\frac{F_{L}}{F_{t h}}\right) \quad---(1) \\
& \Delta d \sim \frac{A\left(F_{L}-F_{t h}\right)}{\rho L_{v}} \quad---(2) .
\end{aligned}
$$

Eq. (1) is known as so-called "Beer's law" which expresses a logarithmic dependence of ablation depth per pulse $(\Delta d)$ on the laser fluence $\left(F_{L}\right) . F_{t h}$ is the threshold fluence for ablation and $\alpha$ is the absorption coefficient of the target. The ablation depth is normally well fitted by this equation under short-pulse (femto- /pico-second pulse duration) irradiation condition where thermal conduction into the target can be neglected. The result obtained from 20 pico-sec laser irradiation [11] is also shown together with present results. In the case of pico-sec laser, the result was well reproduced by this equation. 
For the longer pulse ( tens nano-second pulse duration) irradiation condition, it needs to take the application of another Eq. (2) into account because thermal wave propagation due to the excitation of the lattice vibration is no longer ignored in such time scale [14]. Here, $A$ is a dimensionless coefficient, $\rho$ and $L_{v}$ are the mass density and latent heat per unit mass of the target, respectively. Since the 4-ns pulse duration could be assumed the intermediate between these conditions, both two equations were applied to present results.

As seen in Fig. 1, both Eqs. (1) and (2) were applicable to the pure graphite sample. On the other hand, one can find that experimental result of the deposition sample was well reproduced by Eq. (2) rather than Eq. (1), i.e. Beer's curve deviated from the experimental data in low fluence range. The result indicated that the thermal conduction into the target could not be negligible in this pulse-duration.

All fitting parameters are summarized in table 1. The ablation threshold of the deposition sample was smaller than that of the graphite sample. It could be attributed to the difference of thermal conductivity between deposited layer and pure graphite. According to Ishimoto et al., the thermal conductivity of the deposited layer on JT-60U inner divertor tile was roughly $1 / 30$ of that of ETP-10 graphite [15], hence surface of the deposited layer could be easily heated up during laser irradiation compared to the graphite surface. The absorption coefficients $\alpha$ were $7.4 \mu \mathrm{m}^{-1}$ for the deposited sample and $8.9 \mathrm{\mu m}^{-1}$ for the graphite sample. Those values were smaller than that obtained from the 20 pico-sec laser irradiation by a factor of $\sim 4$. On the other hand, those were larger than that obtained from 25 nano-sec ArF $(193 \mathrm{~nm})$ laser irradiation reported by Shu et al. [5] by a factor of $\sim 4$. It means that pulse duration can play important role from the viewpoint of removal efficiency. From the present result, the removal rate of nano-sec laser seems to be better than that of pico-sec laser. Meanwhile, we have to carefully see the behaviour of removed species as well as removal efficiency, which is discussed in the next section.

\subsection{Emitted ions and desorbed gases analyses}

Fig. 2 (a) shows the visible emission spectrum obtained from the deposited sample during the laser 
irradiation with $F_{L}=1.0 \mathrm{~J} / \mathrm{cm}^{2}$, which was the highest fluence in this study. There were only peaks from $\mathrm{C}_{2}$ swan band $\left(\mathrm{d}^{3} \Pi_{g}-\mathrm{a}^{3} \Pi_{u}\right)$ emission, for example 469.7, 516.5 and $589.9 \mathrm{~nm}$, and no emissions from $\mathrm{C}$, $\mathrm{C}^{+}$or $\mathrm{C}^{2+}$ even at this fluence. Mass distributions of the emitted ions measured by TOFMS are shown in Fig. 2 (b). In the case of deposition sample, the emission of large carbon-clusters was dominant and few $\mathrm{C}^{+}$or $\mathrm{C}^{2+}$ emission was observed, which was consistent with the result of visible light observation. $\mathrm{H}^{+}$ emission was observed only in the deposition sample in the fluence range of $F_{L}>0.7 \mathrm{~J} / \mathrm{cm}^{2}$. One can note that the size of the carbon-cluster emitted from the deposited sample tended to be larger than that from the graphite sample.

Fig. 3 shows the typical mass distribution of the desorbed gases measured by QMS. After start of the laser irradiation, $m / e=2\left(\mathrm{H}_{2}\right), 16\left(\mathrm{CH}_{4}\right), 26\left(\mathrm{C}_{2} \mathrm{H}_{2}\right), 28\left(\mathrm{CO}+\mathrm{C}_{2} \mathrm{H}_{4}\right), 44\left(\mathrm{CO}_{2}\right)$ and $50\left(\mathrm{C}_{4} \mathrm{H}_{2}\right)$ peaks appeared (Fig.3 (b)), whereas there was only $m / e=18\left(\mathrm{H}_{2} \mathrm{O}\right)$ peak before the irradiation (Fig.3 (a)). Here, the relation between $\mathrm{H}_{2}$ and hydrocarbon or $\mathrm{H}_{2} \mathrm{O}$ production is important because, as mentioned before, the removal of tritium as hydrogen gas is more desirable than that as hydrocarbon or tritiated water. $\mathrm{H}_{2} \mathrm{O}$ signal intensity showed no significant increase even after the laser irradiation started, i.e. hydrogen was released not as water but as hydrogen gas or hydrocarbon in this experiment. Major hydrocarbon species emitted here were $\mathrm{C}_{2} \mathrm{H}_{2}(m / e=26)$ and $\mathrm{C}_{2} \mathrm{H}_{4}(m / e=28)$. Since it is difficult to divide $m / e=28$ peak into $\mathrm{C}_{2} \mathrm{H}_{4}$ and $\mathrm{CO}, \mathrm{C}_{2} \mathrm{H}_{4}$ is not pertinent for the evaluation. Therefore, we focused only on the relation between $\mathrm{C}_{2} \mathrm{H}_{2}$ and $\mathrm{H}_{2}$. Fig. 4 (a) shows the signal intensity ratio $\mathrm{I}\left[\mathrm{C}_{2} \mathrm{H}_{2}\right] / \mathrm{I}\left[\mathrm{H}_{2}\right]$ as a function of laser fluence. The ratio increased monotonically with increasing the laser fluence.

According to our past investigations using pico-sec laser with $266 \mathrm{~nm}$ wavelength, $\mathrm{I}\left[\mathrm{C}_{2} \mathrm{H}_{2}\right] / \mathrm{I}\left[\mathrm{H}_{2}\right]$ continuously increased up to a certain laser fluence as well as the present result. This was probably attributed to the fact that $\mathrm{C}_{2} \mathrm{H}_{2}$ is thermodynamically stable even at high temperature, whereas most of hydrocarbon species are unstable above $1000 \mathrm{~K}$. After a threshold fluence, however, $\mathrm{I}\left[\mathrm{C}_{2} \mathrm{H}_{2}\right] / \mathrm{I}\left[\mathrm{H}_{2}\right]$ turned to decreasing with the laser fluence. We defined each fluence range below and above this threshold as "weak"-ablation range (WAR) and "strong"-ablation range (SAR), respectively [10-12]. In 
SAR, visible light emissions from $\mathrm{C}^{+}$and $\mathrm{C}^{2+}$ were observed, and $\mathrm{C}^{+}$or $\mathrm{C}^{2+}$ ions were dominant in TOFMS spectrum, i.e. SAR was associated with ionization of carbon. In such a high fluence range, breaking $\mathrm{C}-\mathrm{H}$ bond $(4.5 \mathrm{eV}) / \mathrm{C}-\mathrm{C}$ bond $(3.6 \mathrm{eV})$ by $266 \mathrm{~nm}$-photon $(4.7 \mathrm{eV})$ and ionization of carbon $(11.3 \mathrm{eV})$ by three-photon ionization became pronounced, which could inhibit the production of $\mathrm{C}_{2} \mathrm{H}_{2}$. Eventually, we concluded laser irradiation in SAR was better for the removal of codeposition with minimizing the hydrocarbon production. By analogy with that result, the fluence range in the present study was still WAR, which was not sufficient to reduce the hydrocarbon production. Fig. 4 (b) shows the ratio between the number of the desorbed hydrogen as $\mathrm{H}_{2}$ by 150 shots of irradiation and that of hydrogen retained in the ablated volume: $\mathrm{H}_{\text {desorbed as } \mathrm{H} 2} / \mathrm{H}_{\text {retained. }}$ In this study, the maximum ablation depth per pulse was $\sim 200 \mathrm{~nm}$, hence 150 shots irradiation corresponds to the removal of almost whole layer $(\sim 30 \mu \mathrm{m})$ in the case of highest laser fluence. $\mathrm{H}_{\text {retained }}$ was estimated from the ablated volume and $\mathrm{H}$ concentration in the deposited layer. The ablated volume was calculated from the measured ablation depth and the laser-spot size assuming a parabolic radial ablation profile. As mentioned above, hydrogen was retained homogeneously in the co-deposited layer with the nearly constant H/C ratio of $\approx$ 0.03 [13] which corresponds to the $\mathrm{H}$ concentration of $\approx 1.4 \times 10^{27} \mathrm{H} / \mathrm{m}^{3}$. Although the data points from the nano-sec laser are relatively scattered compared to the result from the pico-sec laser irradiation, one can say that $\mathrm{H}_{\text {desorbed as } \mathrm{H} 2} / \mathrm{H}_{\text {retained }}$ of nano-sec laser irradiation was more or less $\sim 0.4$ in this experimental fluence range. This means that roughly $40 \%$ of retained hydrogen was released as $\mathrm{H}_{2}$ form in these irradiation conditions whereas another $60 \%$ was released as other chemical forms such as water or hydrocarbon species. Since $\mathrm{H}_{2} \mathrm{O}$ partial pressure did not increase during the irradiation as seen in QMS spectrum (see Fig. 3), the remaining $60 \%$ of hydrogen was likely to be released as hydrocarbon species. Higher-power irradiation should be performed in future work in order to confirm whether SAR appears also in such nano-sec laser condition or not. 


\section{Summary and outlook}

Removal of carbon deposition on JT-60 divertor tile by LID using the fourth-harmonic emission $(266 \mathrm{~nm})$ of a 4 nano-sec pulsed Nd:YAG laser was demonstrated. The ablation depth per laser fluence showed a linear dependence on the laser fluence rather than a logarithmic dependence, whereas the ablation depth by pico-sec laser irradiation showed good logarithmic dependence on the laser fluence in the previous study. This means the thermal conduction into the target could not be negligible in such nano-sec laser condition. The ablation threshold of the deposited layer was slightly lower than that of the graphite sample, which could be attributed to the difference in thermal conductivity between deposited layer and pure graphite. The removal rate of the nano-sec laser was higher than that of pico-sec laser in the fluence range of $<\sim 0.5 \mathrm{~J} / \mathrm{cm}^{2}$. On the other hand, the production ratio I[hydrocarbon species] / I $\left[\mathrm{H}_{2}\right]$ continuously increased with the laser fluence, and we could not observe the strong ionization of carbon in this experiment. This indicated that the fluence range in this study was still "weak"-ablation range, which was not sufficient to reduce the hydrocarbon production. Higher power irradiation is required in order to confirm whether "strong"-ablation range, where hydrogen is released as hydrogen gas with minimizing the hydrocarbon production, appears in the nano-sec laser condition, which is the future work. 


\section{References}

[1] ITER Physics Expert Group, ITER physics basis, Nucl. Fusion 39 (1999) 2137-2664

[2] G. Federici, R.A. Anderl, P. Andrew, J.N. Brooks, R.A Causey, J.P. Coad, D. Cowgill, R.P. Doemer, A.A. Haasz, G. Janeschitz, W. Jacob, G.R. Longhurst, R. Nygren, A. Peacock, M.A. Pick, V. Philipps, J. Roth, C.H. Skinner, W. R. Wampler, J. Nucl. Mater. 266-269 (1999) 14.

[3] C. H. Skinner, C. A. Gentile, A. Carpe, G. Guttadora, S. Langish, K. M. Young, W. M. Shu and H. Nakamura, J. Nucl. Mater. 301 (2002) 98

[4] C. H. Skinner, N. Bekris, J. P. Coad, C. A. Gentile and M. Glugla, J. Nucl. Mater. 313-316 (2003) 496

[5] W. M. Shu, Y. Kawakubo, K. Masaki, M.F. Nishi, J. Nucl. Mater. 313-316 (2003) 584

[6] F. Le Guern, C. Hubert, S. Mousset, E. Gauthier, C. Blanc, P. Wodling and J.M. Weulersse, J. Nucl. Mater. 335 (2004) 410

[7] T. Åkermark, B. Emmoth and H. Bergsåker, J. Nucl. Mater. 359 (2006) 220

[8] Ch. Grisolia, A. Semerok, J. M. Weulersse, F. Le Guern, S. Fomichev, F. Brygo, P. Fichet, P. Y. Thro, P. Coad, N. Bekris, M. Stamp, S. Rosanvallon, G. Piazza, J. Nucl. Mater. 363-365 (2007) 1138

[9] Y. Sakawa, T. Shibahara, K. Sato, T. Tanabe, J. Plasma and Fusion Research Series 7 (2006) 138.

[10] Y. Sakawa, K. Sato, T. Shibahara, T. Tanabe, Fusion Eng. Des. 81 (2006) 381.

[11] D. Watanabe, Y. Sakawa, T. Shibahara, K.Sugiyama, T. Shoji, K. Yamazaki, T. Tanabe, J. Nucl. Mater. 363-365 (2007) 972-976

[12] Y. Sakawa, D. Watanabe, T. Shibahara, K. Sugiyama, T. Tanabe, J. Nucl. Mater. 367-370 (2007) 1516

[13] Y. Hirohata, T. Shibahara, T. Tanabe, T. Arai, Y. Gotoh, Y. Oya, H. Yoshida, Y. Morimoto, J. Yagyu, K. Masaki, K. Okuno, T. Hino, N. Miya, J. Nucl. Mater. 337-339 (2005) 609Y. Gotoh, T. Arai, J. Yagyu, K. Masaki, K. Kodama, N. Miya, J. Nucl. Mater. 329-333 (2004) 840

[14] A. Amoruso, R. Bruzzese, N. Spinelli, and R. Velotta, J. Phys. B, At. Mol. Opt. Phys. 32 (1999) R131

[15] Y. Ishimoto, Y. Gotoh, T. Arai, K. Masaki, N. Miya, N. Oyama and N. Asakura., J. Nucl. Mater. 350 (2006) 301-309 
table 1

Summary of fitting parameters for equations (1) and (2). Parameters for 20 pico-sec (266 nm) and 25 nano-sec $(193 \mathrm{~nm})$ lasers were from references [11] and [5], respectively.

\begin{tabular}{lccccc}
\hline \multirow{2}{*}{ Sample } & \multicolumn{2}{c}{ Equation (1) } & & \multicolumn{2}{c}{ Equation (2) } \\
\cline { 2 - 3 } \cline { 5 - 6 } & $\mathrm{F}_{\text {th }}\left[\mathrm{J} / \mathrm{cm}^{2}\right]$ & $\alpha\left[\mu \mathrm{m}^{-1}\right]$ & & $\mathrm{F}_{\text {th }}\left[\mathrm{J} / \mathrm{cm}^{2}\right]$ & $\mathrm{A}$ \\
\hline Deposition & 0.28 & 7.4 & & 0.13 & $1.3 \times 10^{6}$ \\
Graphite (ETP-10) & 0.37 & 8.9 & & 0.29 & $8.9 \times 10^{5}$ \\
Deposition / 20 Pico-sec laser [11] & 0.18 & 35 & & & \\
Deposition / 25 nano-sec laser [5] & 1.0 & 1.9 & & & \\
\hline
\end{tabular}




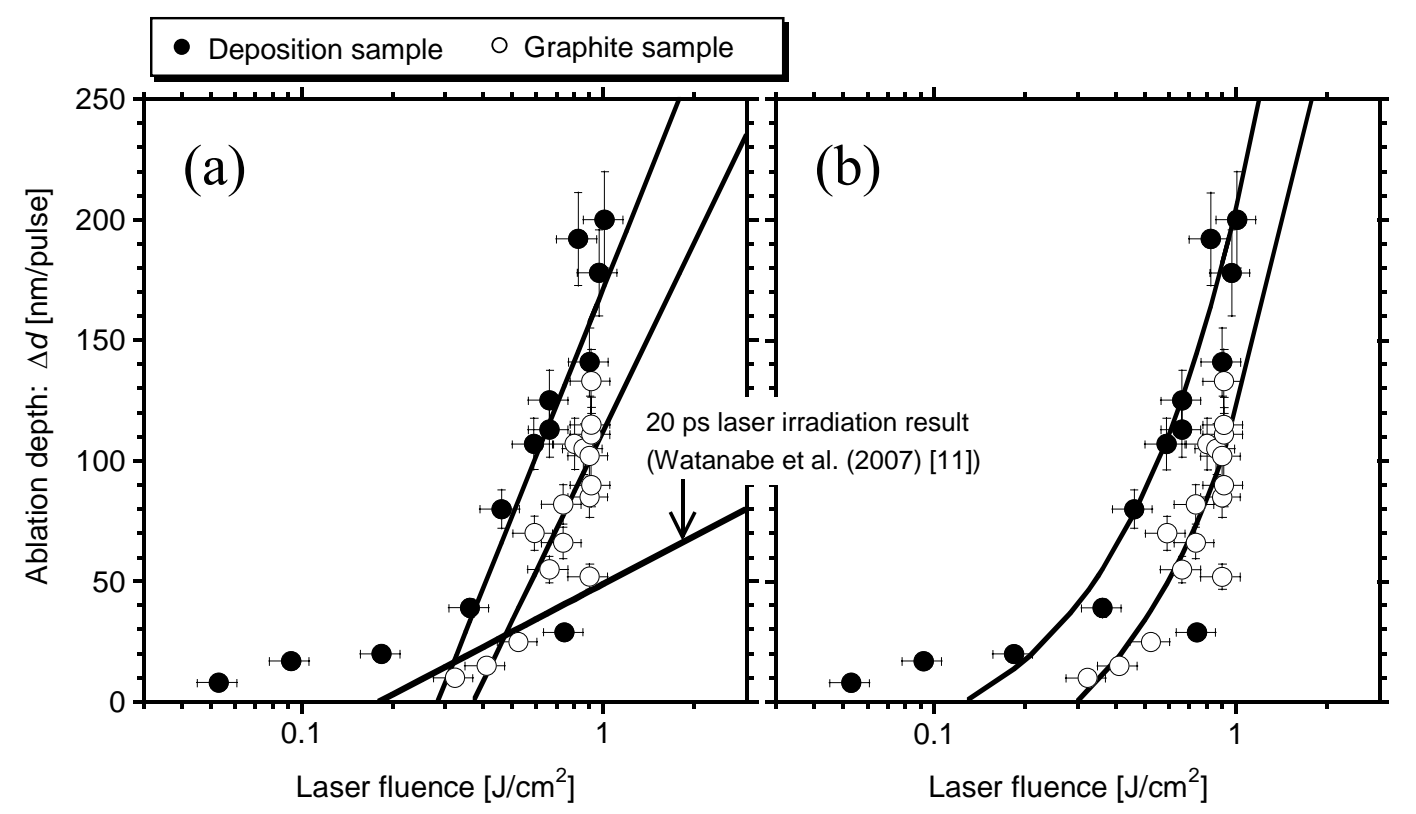

Figure 1

Ablation depth per pulse $\Delta d$ fitted by (a) equation (1) and (b) equation (2). The result obtained from pico-sec laser irradiation (also for similar graphite with deposition sample) [11] is shown in (a) together with the present result. 

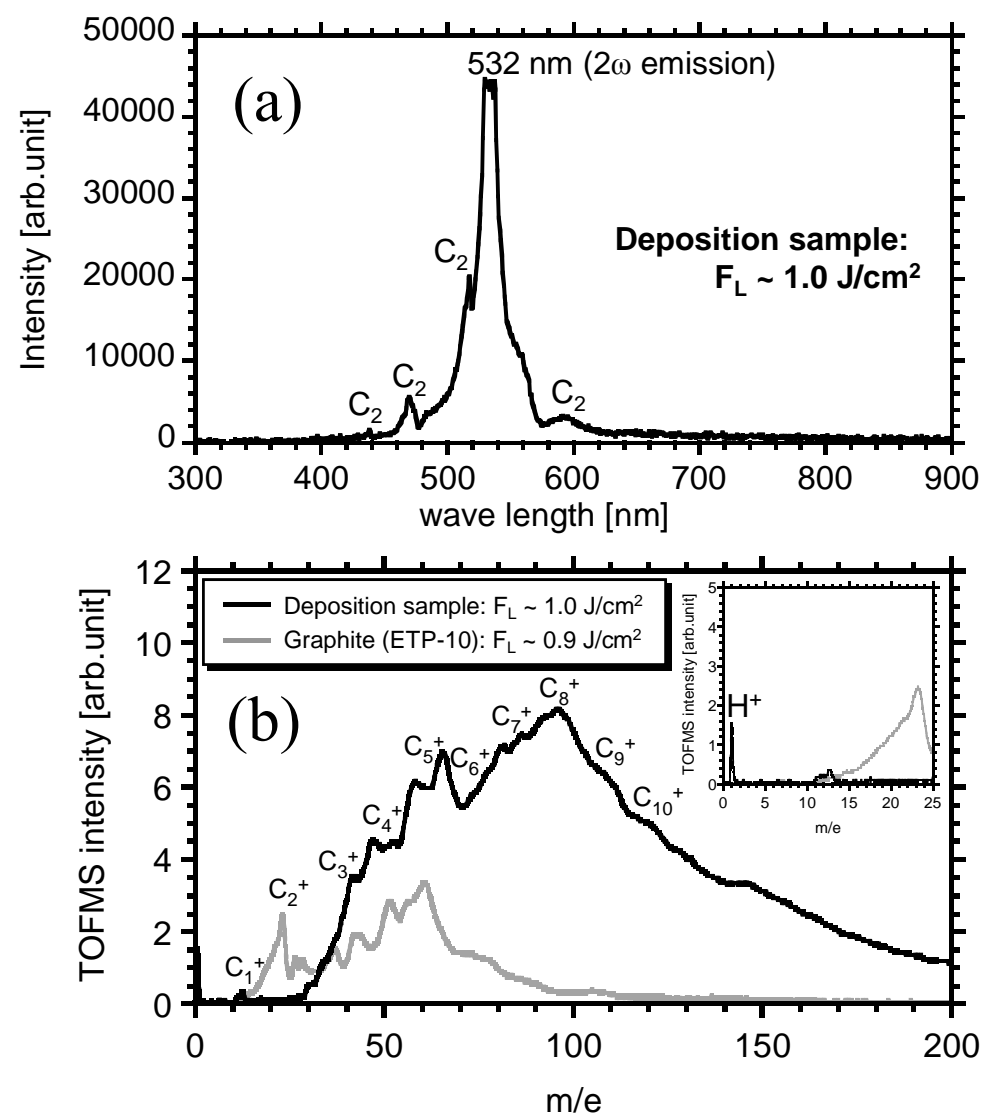

Figure 2

(a) Visible emission spectrum obtained from the deposited sample with the fluence of $1.0 \mathrm{~J} / \mathrm{cm}^{2}$. (b) TOFMS spectra obtained from the deposition $\left(\mathrm{F}_{\mathrm{L}} \sim 1.0 \mathrm{~J} / \mathrm{cm}^{2}\right)$ and graphite $\left(\mathrm{F}_{\mathrm{L}} \sim 0.9 \mathrm{~J} / \mathrm{cm}^{2}\right)$ samples. Both are the first scan after irradiation started. Carbon tended to be released as larger cluster in the case of deposition sample. 


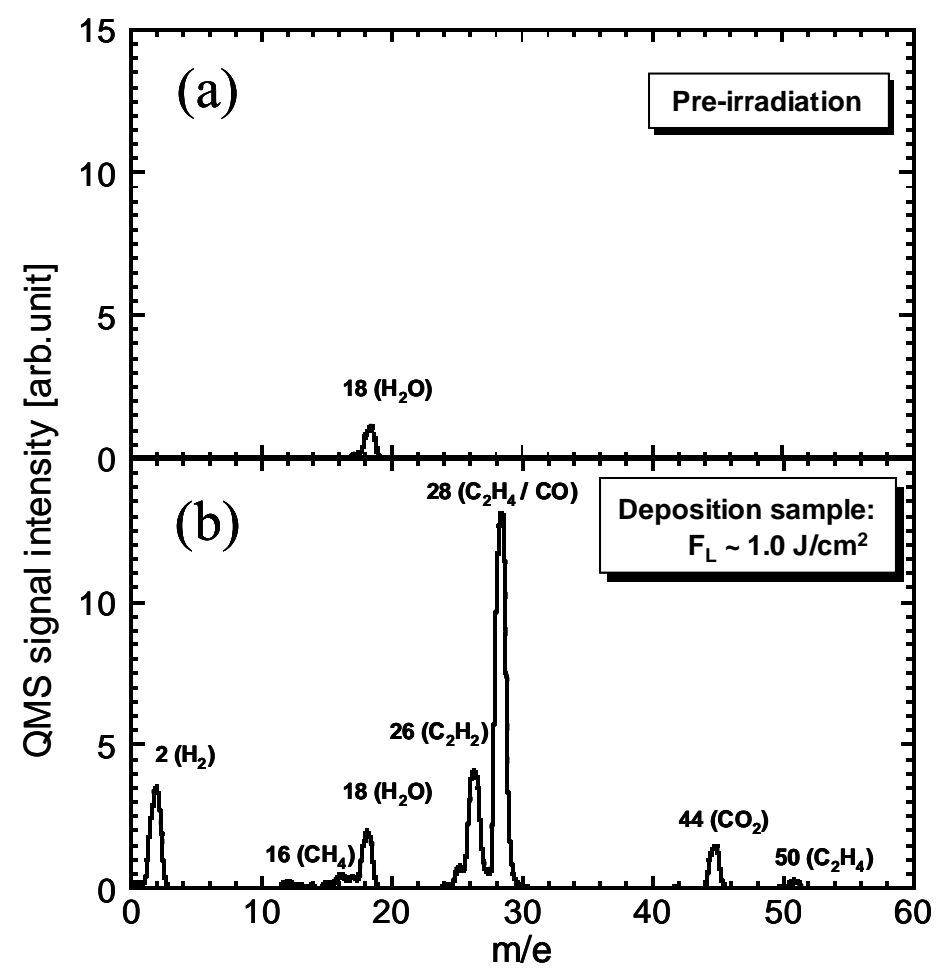

Figure 3

Typical mass distributions of the desorbed gases measured by QMS. The spectra were obtained from (a) before the irradiation started (reference), and (b) during the irradiation to the deposition sample with the fluence of $1.0 \mathrm{~J} / \mathrm{cm}^{2}$. 


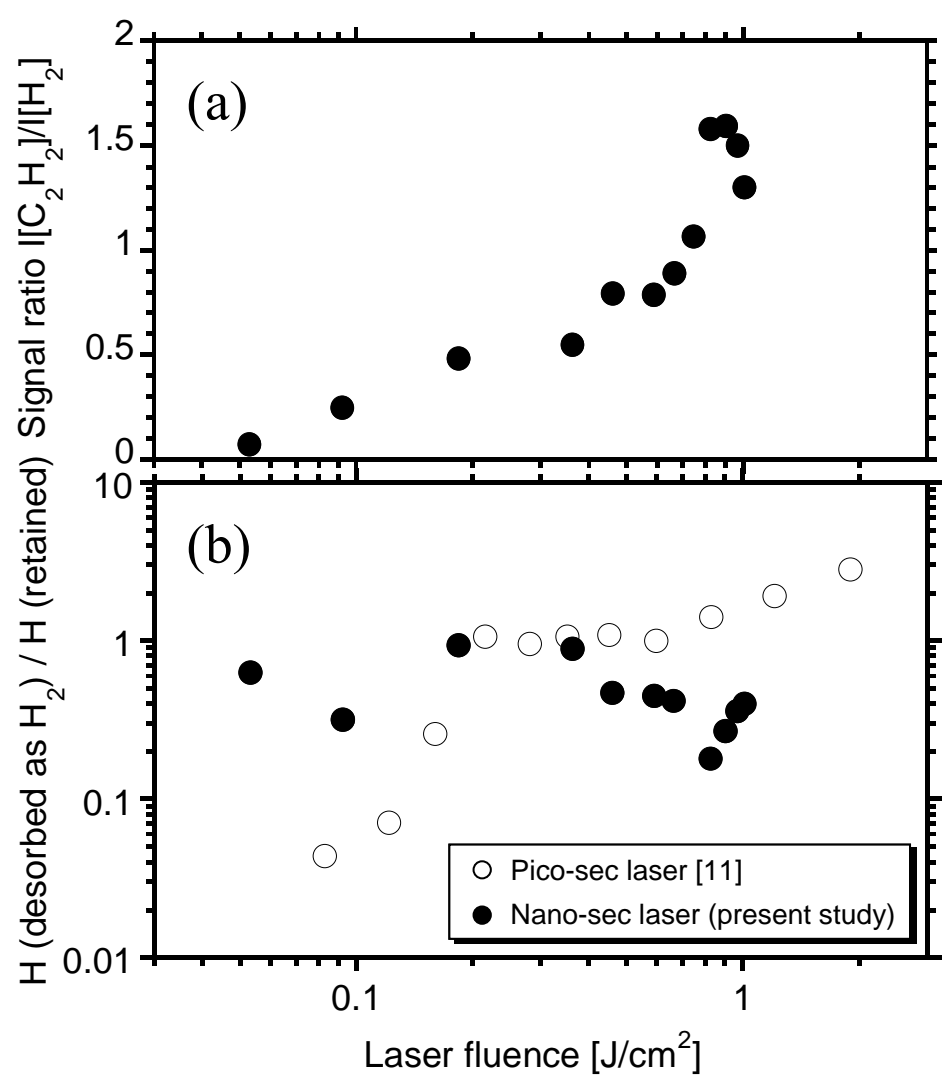

Figure 4

(a) Signal intensity ratio: $\left[\mathrm{C}_{2} \mathrm{H}_{2}\right] /\left[\mathrm{H}_{2}\right]$ as a function of laser fluence. The ratio kept increasing in this fluence range. (b) The ratio between the number of the desorbed hydrogen as $\mathrm{H}_{2}$ and that of hydrogen retained in the ablated volume: $\mathrm{H}_{\text {desorbed }} / \mathrm{H}_{\text {retained }}$ as a function of laser fluence. 